\title{
miR-128 and miR-149 enhance the chemosensitivity of temozolomide by Rap1B-mediated cytoskeletal remodeling in glioblastoma
}

\author{
XIAOLING SHE ${ }^{1,2}$, ZHIBIN YU $^{2,3}$, YULONG CUI $^{1}$, QIANQIAN LEI ${ }^{2,3}$, ZEYOU WANG $^{2,3}$, \\ GANG XU' ${ }^{2,3}$, JUANJUAN XIANG ${ }^{2}$, MINGHUA WU ${ }^{2,3}$ and GUIYUAN LI ${ }^{2}$
}

\begin{abstract}
${ }^{1}$ Second Xiangya Hospital, Central South University, Changsha, Hunan $410011 ;{ }^{2}$ Hunan Cancer Hospital and the Affiliated Tumor Hospital of Xiangya Medical School, Cancer Research Institute, Central South University, Changsha, Hunan 410013; ${ }^{3}$ The Center for Skull Base Surgery and Neurooncology, Changsha, Hunan 410008, P.R. China
\end{abstract}

Received February 28, 2014; Accepted May 21, 2014

DOI: $10.3892 /$ or.2014.3318

\begin{abstract}
Glioblastoma multiforme (GBM) is one of the most deadly diseases affecting humans, and is often characterized by poor survival and by high resistance to chemotherapy and radiotherapy. Temozolomide (TMZ) is an oral alkylating agent which is widely used in the treatment of GBM following surgery. Although TMZ may restrain GBM growth, TMZ resistance is also common and accounts for numerous cases of treatment failure. Studies indicate that aberrant miRNA expression is associated with hallmark malignant properties of GBM. Thus, miRNA-based anticancer therapeutic approaches have been exploited, either alone or in combination with standard targeted therapies to enhance the efficacy of chemotherapy agents. In the present study, we demonstrated that the expression of miR-128 and miR-149 was downregulated in glioblastoma, and their overexpression inhibited the invasion of glioblastoma cells by targeting Rap1B-mediated cytoskeletal and related molecular alterations. Moreover, miR-128 and miR-149 enhanced the chemosensitivity of glioblastoma cells to TMZ.
\end{abstract}

\section{Introduction}

microRNAs (miRNAs), small non-coding RNAs that are 20-22 nucleotides in length, play important roles in cancer including proliferation, aggressiveness and development of metastasis (1-3). miRNAs have the ability to modulate key cellular processes that define the cell phenotype, making them highly promising therapeutic targets. Glioblastoma multiforme

Correspondence to: Professor Minghua Wu, Hunan Cancer Hospital and the Affiliated Tumor Hospital of Xiangya Medical School, Cancer Research Institute, Central South University, Changsha, Hunan 410013, P.R. China

E-mail: wuminghua554@aliyun.com

Key words: cytoskeleton, miR-128, miR-149, temozolomide, glioblastoma, F-actin
(GBM) is the most common and aggressive primary brain tumor in adults. Elucidation of the molecular pathogenesis of GBM is crucial to improve the overall survival of patients (4). The current standard therapy for GBM includes surgical resection or biopsy, followed by a combination of radiation (RT) and chemotherapy with temozolomide (5). Temozolomide (TMZ), an alkylating agent, is a cytotoxic antitumor prodrug against malignant brain tumors. At physiological $\mathrm{pH}$, TMZ undergoes decomposition to the active compound MTIC $(6,7)$. Although $\mathrm{TMZ}$ may restrain GBM growth, TMZ resistance is common and accounts for GBM treatment failure (8). Recent analyses have revealed that several miRNAs are clinically implicated in GBM, with some reports indicating the association of the regulation of miRNA expression and the chemosensitivity to TMZ (8-11). For example, expression levels of miR-181b and miR-181c may serve as predictive markers of response to RT/ TMZ therapy in glioblastoma patients (12). Chronic TMZ exposure was found to result in acquired TMZ resistance and elevated miR-21 expression. Concomitant treatment with miR-21 inhibitor and TMZ resulted in a significantly higher apoptosis rather than TMZ treatment alone (8). miR-21 inhibitor combined with TMZ significantly enhanced human glioblastoma stem cell apoptosis (8). miR-125b-2 is overexpressed in glioblastoma multiforme tissues and the corresponding stem cells (GBMSCs); downregulation of miR-125b-2 expression in GBMSCs could allow TMZ to induce GBMSC apoptosis (13). Our previous research found that miR-128, miR-149 and miR-181a/b were significantly downregulated and may be tumor suppressors in astrocytomas (14). Further research validated that miR-181a/b/c/d were significantly downregulated in astrocytomas and glioblastoma of different grades, and their overexpression inhibited the growth and invasion of glioblastoma cells and enhanced the chemosensitivity of TMZ by Rap1B-mediated cytoskeletal remodeling. Rap1B is a member of the Rap1 family, and is a Ras-like GTPase that regulates several basic cellular functions, such as growth, adhesion and migration (15). Rap1B was also found to be a possible negative regulator of miR-128 and miR-149 by a docking study of miRNA/mRNA omics in human grade I-III astrocytomas (14). Recent studies suggest that miR-128 and miR-149 play an 
important role in various diseases, including the progression of malignant tumors. miR-128 is a brain-enriched miRNA, which has tissue-specific and developmental-specific expression patterns, mainly in neurons rather than in astrocytes (16). Downregulation of miR-128 has been reported in several cancer types, for example, neuroblastoma (17) glioblastoma $(14,18,19)$ prostate $(20)$, lung cancer $(21)$ and MLL-AF4 acute lymphocytic leukemia (22). miR-128 exerted a pro-apoptotic effect in a p53 transcription-dependent and -independent manner via the PUMA-Bak axis in HEK293T cells (human embryonic kidney), HCT116 cells (human colon carcinoma cells) and MCF-7 cells (human breast adenocarcinoma), and miR-128 regulated the chemotherapeutic sensitivity of breast tumor-initiating cells via Bmi-1 and ABCC5 (23). The overexpression of miR-128 was found to suppress p70S6K1 and expression of its downstream signaling molecules HIF-1 and VEGF, and to attenuate cell proliferation, tumor growth and angiogenesis (24).

Recent studies have revealed the essential role of miR-149 in various types of diseases, including the progression of various types of malignant tumors. However, the results are controversial, and miR-149 has been shown to function as both a tumor suppressor (25) and as an oncogene (26) in the development of multiple types of solid tumors. p53 directly upregulates miR-149 which in turn targets glycogen synthase kinase-3 $\alpha$, resulting in increased expression of Mcl-1 and resistance to apoptosis in melanoma cells (26). miR-149 was found to be significantly downregulated in GC cell lines and clinical samples in comparison to normal gastric epithelial cells and adjacent non-tumor tissues, respectively (27). miR-149 suppresses tumor cell motility, and the pre-miR-149 polymorphism may affect the processing of miR-149, resulting in a change in the abundance of the mature form of miRNA, which, in turn, modulates tumor progression and patient survival (28). miR-149 was found to be downregulated and to function as a tumor suppressor by inhibiting oncogene expression, and it inhibited the proliferation and invasion of glioma cells by blocking AKT1 signaling in glioblastoma (29).

In the present study, we further confirmed changes in the expression of miR-128 and miR-149 in astrocytomas of different grades (WHO I, II and III) and glioblastoma (WHO IV), and we investigated the effect of miR-128 and miR-149 on cellular proliferation, invasion and its functional mechanism by targeting Rap1B. We also demonstrated that miR-128 or miR-149 enhanced the chemosensitivity of temozolomide by Rap1B-mediated cytoskeletal remodeling in glioblastoma.

\section{Materials and methods}

Samples. Human glioma-derived cell lines U251 and U87 were obtained from the Cell Center of the Peking Union Medical College in China. All human primary brain tumor samples were obtained from randomly selected cancer patients at the Second Affiliated Hospital of Xiangya, China, and all of the diagnoses were pathologically confirmed. Written informed consent was obtained from each patient who participated in the study before surgery, and all of the protocols were reviewed by the Joint Ethics Committee of the Central South University Health Authority and performed in accordance with national guidelines.
Cell culture. The U251 and U87 cells were grown in Dulbecco's modified Eagle's medium (DMEM) with $10 \%$ fetal calf serum (FCS) and standard antibiotics. All cells were maintained at $37^{\circ} \mathrm{C}$ under an atmosphere of $5 \% \mathrm{CO}_{2}$ and $95 \%$ air.

RNA extraction and real-time PCR. Real-time PCR was carried out as previously described (30). Total RNA was extracted using TRIzol ${ }^{\circledR}$ reagent (Invitrogen, Carlsbad, CA, USA) from samples. Real-time PCR reactions were performed using a qSYBR-Green-containing PCR kit (Invitrogen) and human $\beta$-actin or U6 snRNA was used as an endogenous control for mRNA or miRNA detection, respectively. Expression of each gene was quantified by measuring $\mathrm{Ct}$ values and normalized using the $2^{-\Delta \Delta c t}$ method relative to U6 snRNA or $\beta$-actin.

Luciferase assay. The 3'-untranslated regions (UTRs) of the Rap1B gene were synthesized, annealed, and then inserted into the pMIR-REPORT ${ }^{\mathrm{TM}}$ luciferase vector (Ambion) using the HindIII (aagctt) and SpeI (actagt) sites located downstream from the stop codon of luciferase. There was one region of the Rap1B gene 3'-UTRs for miR-128 and miR-149 in TargetScan 5.2 software. The Luc-wt or Luc-mut plasmid was co-transfected with miR-128 or miR-149 mimics into U251 cells, and the pMIR-REPORT $\beta$-galactosidase control vector was transfected as a control. Firefly luciferase activity was measured consecutively using the luciferase assay system (Promega) $48 \mathrm{~h}$ after transfection. All the experiments were performed at least three times.

Protein extraction and western blotting. Protein extraction and western blot analysis were performed as previously described (30). Rabbit polyclonal primary antibodies against Rap1B, Cdc42, RhoA and N-cadherin were purchased from Cell Signaling Technology (Beverly, MA, USA), and mouse monoclonal antibodies against GAPDH and $\alpha$-tubulin were purchased from Santa Cruz Biotechnology, Inc. (Santa Cruz, CA, USA).

Colony formation assay. Synthesized RNA duplexes of scrambled miRNAs, miR-128 and miR-149 mimics were obtained from GeneChem (Shanghai, China). Temozolomide (T-2706; RD, USA) was obtained from Sigma. Cells were seeded at $1 \times 10^{3}$ cells/well in 6-well plates and transfected with miR-128 or miR-149 mimics followed by treatment with $100 \mu \mathrm{M}$ TMZ after transfection. Two weeks later, cells were fixed in methanol for $30 \mathrm{~min}$ and stained with $1 \%$ crystal violet dye, and the number of colonies was counted. Experiments were repeated three times, and values are expressed as mean and standard deviation.

Cell viability assay. Cell viability was determined by the MTT assay as previously described (30). Briefly, 1,500 cells/well were seeded into 96 -well plates and were treated by miR-128 or miR-149 mimic transient transfection and/or TMZ administration, and MTT (Sigma-Aldrich, St. Louis, MO, USA) assays were performed daily at different times. In this assay, the medium was replaced with fresh medium containing $0.5 \mathrm{mg} /$ $\mathrm{ml}$ MTT for $4 \mathrm{~h}$ and then carefully removed. Subsequently, $150 \mu \mathrm{l}$ of dimethyl sulfoxide (DMSO) was added to each well and mixed for $10 \mathrm{~min}$, and the optical density of each well was 

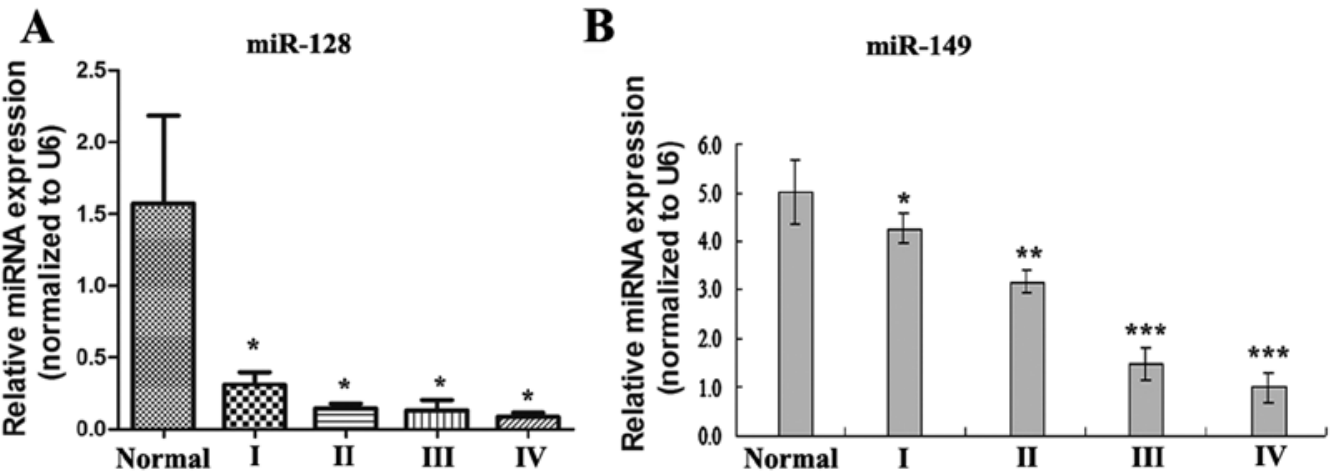

Figure 1. Expression of miR-128 and miR-149 in astrocytomas and normal brain tissues. (A) The expression of miR-128 was decreased in astrocytomas as compared with that in the normal brain tissues, but did not gradually decrease with pathological progression in WHO grade astrocytomas. (B) The expression of miR-149 gradually decreased with pathological progression in different WHO grade astrocytomas.

determined with a scanning multi-well spectrophotometer at a wavelength of $490 \mathrm{~nm}$. The experiment was repeated three times; six parallel samples were measured each time.

Wound closure assay. The cells were transfected with the synthetic miR-128 or miR-149 mimics and grown to $90 \%$ confluency in a 6-well dish. A wound was created using a sterile $10-\mu \mathrm{l}$ pipette tip followed by a wash with $1 \mathrm{X}$ phosphatebuffered saline (PBS) to remove detached cells. Then, the cells were cultured in medium with $2 \%$ serum, and migration at the corresponding wound site was documented using a microscope (Nikon) at different time-points (0, 12, 24 and $48 \mathrm{~h}$ ).

Transwell migration assay. The Transwell migration assay was performed as previously described (18).

Immunofluorescence. U251 cells were transfected with miR-128 or miR-149 mimics, Rap1B siRNA and NC or treatment with TMZ, respectively. The cells were fixed with $4 \%$ paraformaldehyde in PBS for $20 \mathrm{~min}$, permeabilized in $0.25 \%$ Triton X-100 and blocked with 5\% BSA in PBS. Then, cells with or without treatment were stained with FITC-labeled phalloidin (P5282; Sigma, Seelze, Germany) and DAPI (C1002; Beyotime Institute of Biotechnology, Shanghai, China). Images were acquired by laser scanning confocal microscopy (TCSSP5; Leica Microsystems, Wetzlar, Germany).

Statistical analysis. Differences between the variables of the groups were tested using the Student's t-test or one-way ANOVA, using the SPSS 15.0 program. A P-value of $<0.05$ was considered to indicate a statistically significant result.

\section{Results}

miR-128 and miR-149 expression in the astrocytomas of different grades. As shown in Fig. 1, compared to expression in normal brain tissues $(n=8)$, the expression of miR-128 and miR-149 was decreased in most of the primary astrocytomas $(n=20)$, in which the expression of miR-149 gradually decreased with pathological progression in the different WHO grade astrocytomas. However, the expression of miR-128 did not gradually decrease with pathological progression in the different WHO grade astrocytomas. The expression of
miR-128 was not statistically significantly different between grades II, III and IV ( $>>0.05)$, but there was statistically significant differences between grade I and grades II, III and IV astrocytomas $(\mathrm{P}<0.05)$.

Expression of RaplB is negatively regulated by miR-128 and $m i R-149$. As shown in Fig. 2A and B, the miRNA target prediction program TargetScan5.2 was used to identify miR-128 and miR-149 interaction sites in the 3'-UTR of Rap1B. miR-128 and miR-149 mimics had their own binding sites for the 3'-UTR of Rap1B. The luciferase reporter assays showed that miR-128 and miR-149 combined with the sites of the 3'-UTR of Rap1B, respectively. Rap1B is a common bona fide target of miR-128 and miR-149 (Fig. 2C and D). Transfection of the miR-128 and miR-149 mimics resulted in a marked reduction in Rap1B at both the mRNA (Fig. 2E and F) and protein levels (Fig. 2G and $\mathrm{H}$ ) in U251 cells, as compared with negative control (NC) transfection. We also previously evaluated the expression of Rap1B in the astrocytomas tissues and normal brain tissues, and found that the expression of Rap1B was gradually increased with pathological progression in the WHO grade astrocytomas (18). In the present study, we found that the expression of miR-128 or miR-149 and Rap1B was in an inverse trend in the astrocytoma and normal brain tissues. The above data indicate that miR-128 and miR-149 negatively regulate the expression of Rap1B.

TMZ inhibits RaplB expression by upregulating miR-128 and miR-149. As shown in Fig. 3, we observed that TMZ increased the expression of miR-128 and miR-149 by real-time PCR when glioblastoma U251 and U87 cells were treated with $100 \mu \mathrm{M}$ TMZ. Our previous research demonstrated that TMZ also decreased the expression of Rap1B at the mRNA and protein levels by real-time PCR and western blotting (18). Given Rap1B is a common target of miR-128 and miR-149 (Fig. 2), we proposed that TMZ may inhibit Rap1B expression by upregulating miR-128 and miR-149.

miR-128 and miR-149 suppress cell proliferation and invasion, and alter cytoskeletal remodeling by affecting RaplB-associated small GTPase. As shown in Fig. 4A, compared with that of the negative control, miR-128 and miR-149 mimics inhibited cell colony formation in U251 cells 
A

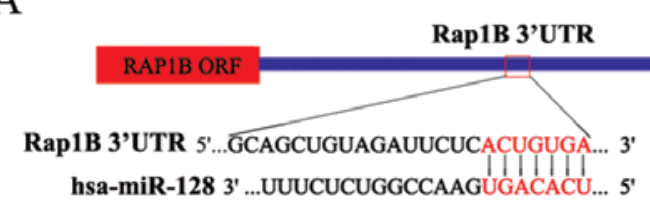

$\mathrm{C}$

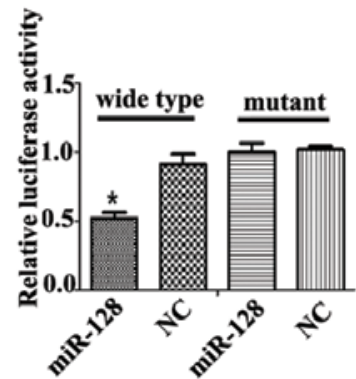

G

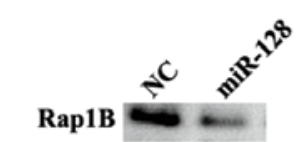

a-tubulin
D
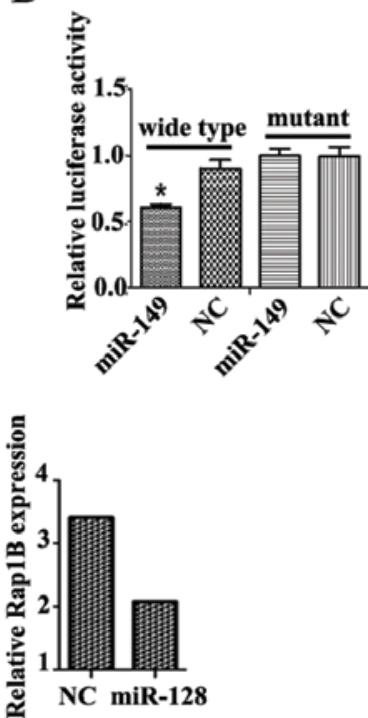

B

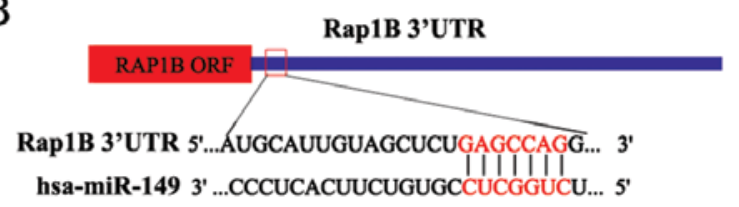

$\mathrm{E}$

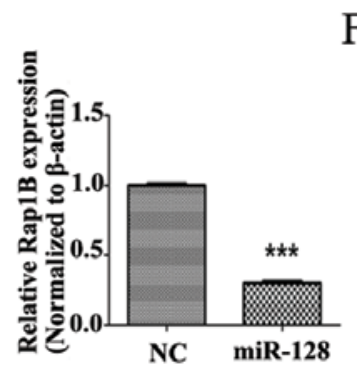

$\mathrm{F}$

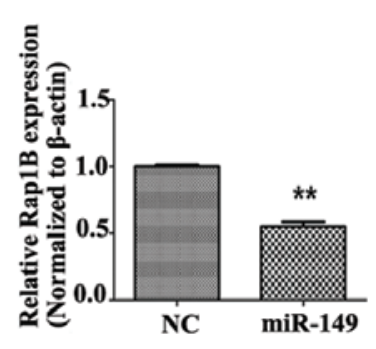

$\mathrm{H}$

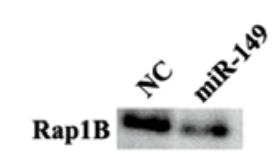

a-tubulin

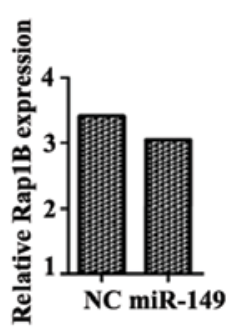

Figure 2. Rap1B is a common target gene of miR-128 and miR-149. (A and B) Schema of the interaction sites of miR-128 and miR-149 and the 3'-UTRs of Rap1B. (C and D) Luciferase assays of U251 glioma cells co-transfected with pMIR-REPORT-WT/mutant 3'-UTR Rap1B and miR-128, miR-149 or negative control as indicated; " $\mathrm{P}<0.05$. (E and F) qRT-PCR showing the mRNA level of Rap1B after miR-128 or miR-149 mimics were transfected into U251 cells for 24 h. miR-128 and miR-149 downregulated the mRNA level of Rap1B; ${ }^{* *} \mathrm{P}<0.01 ;{ }^{* * * *} \mathrm{P}<0.001$. (G and $\mathrm{H}$ ) Western blot analysis showing the protein expression of Rap1B after miR-128 or miR-149 mimics were transfected into U251 cells for $48 \mathrm{~h}$, respectively. miR-128 or miR-149 inhibited the protein expression of Rap1B. $\alpha$-tubulin was used as a loading control.
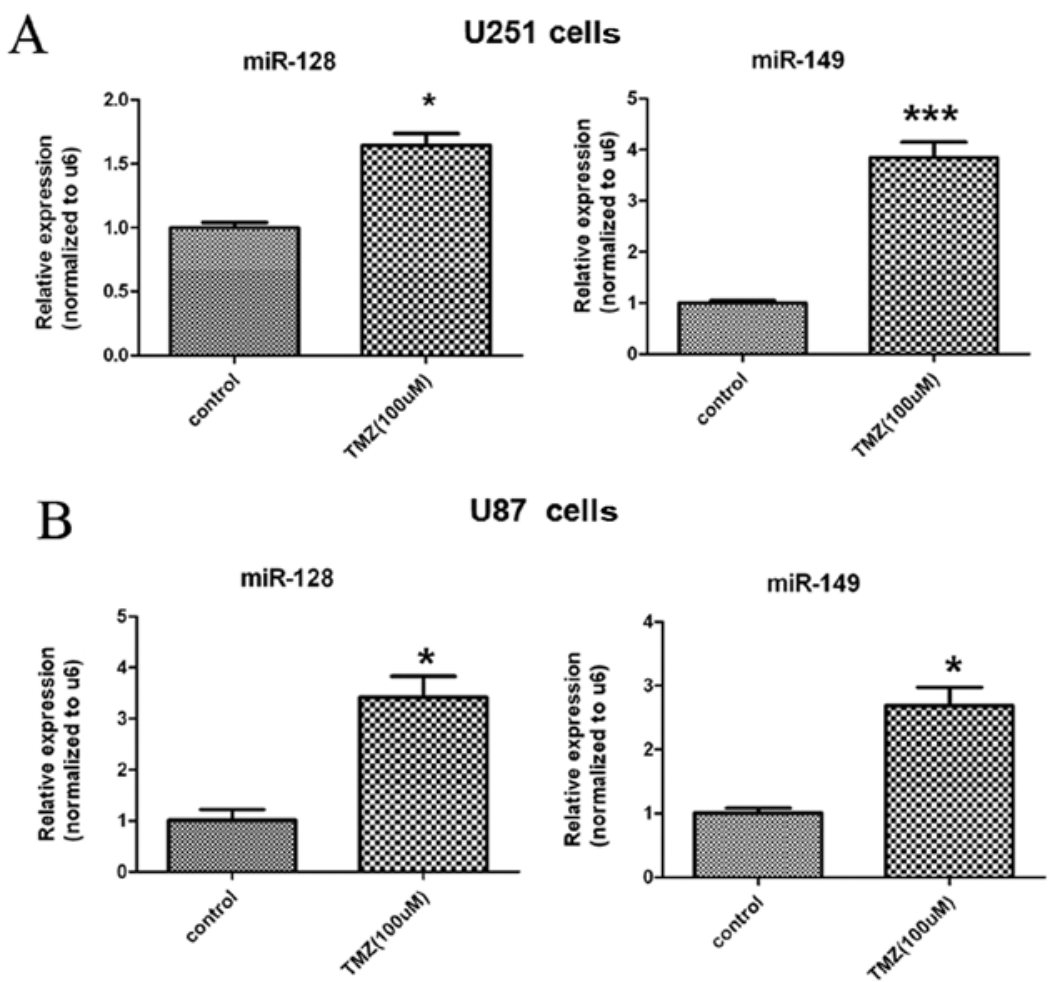

Figure 3. TMZ inhibits Rap1B expression by upregulating miR-128 and miR-149. (A) Real-time PCR showing the expression of miR-128 and miR-149 after U251 cells were treated with $100 \mu \mathrm{M}$ TMZ for $24 \mathrm{~h}$; "P<0.05. (B) Real-time PCR showing the expression of miR-128 and miR-149 after U87 cells were treated with $100 \mu \mathrm{M}$ TMZ for $24 \mathrm{~h} ;{ }^{*} \mathrm{P}<0.05$. 
A

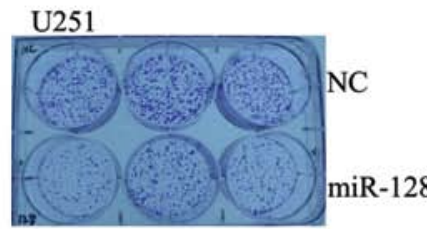

$\mathrm{U} 251$
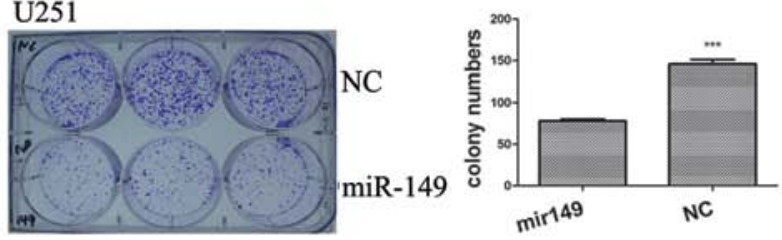

C
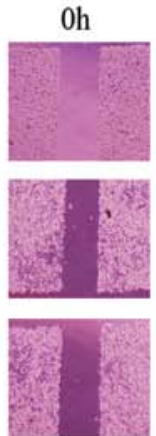

$12 \mathrm{~h}$
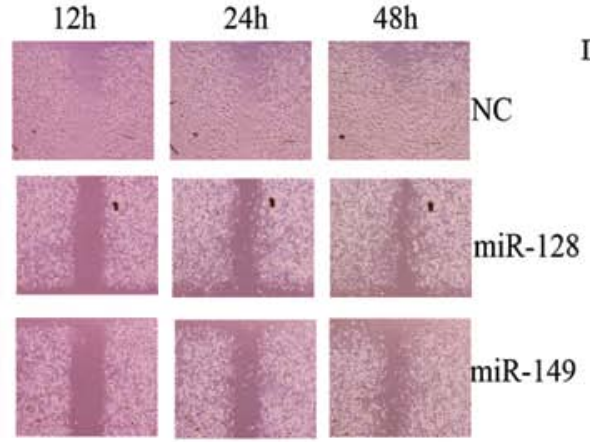

E
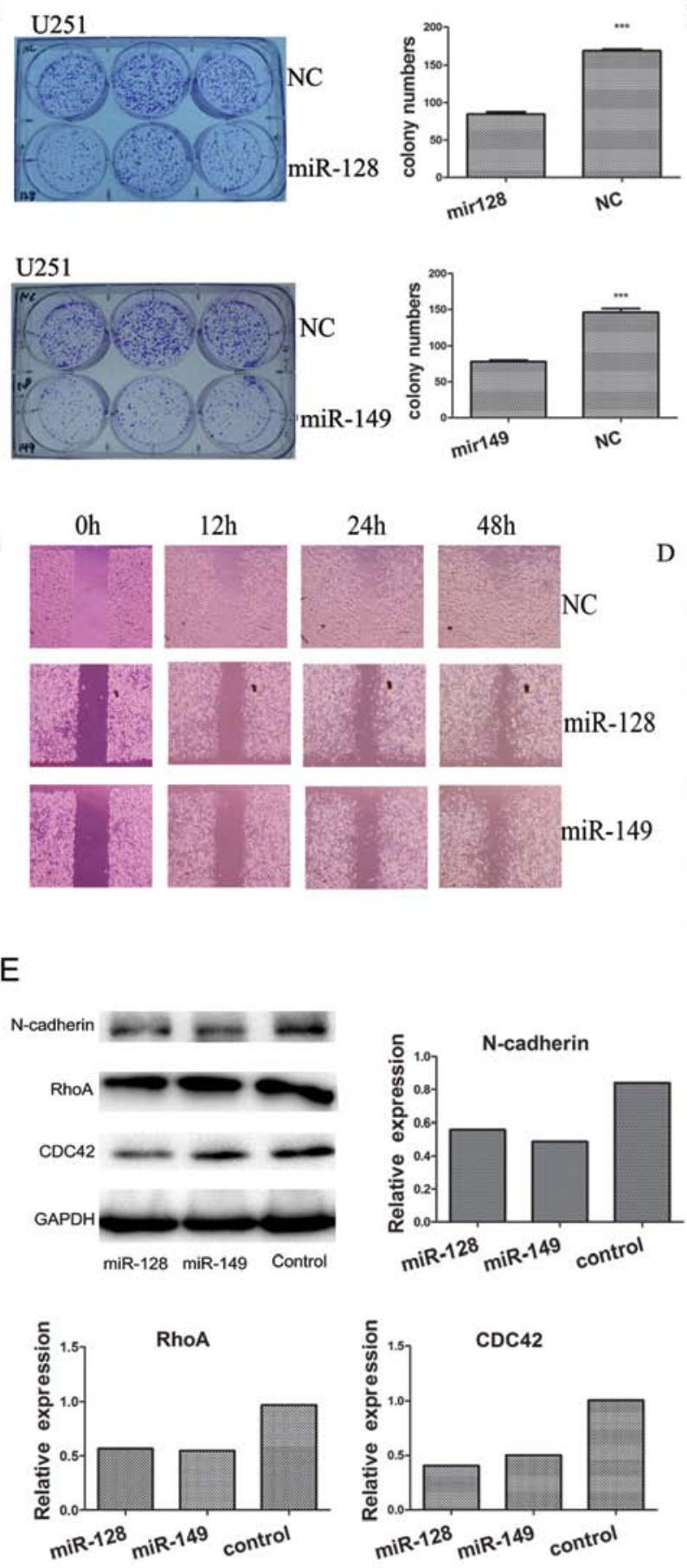

B
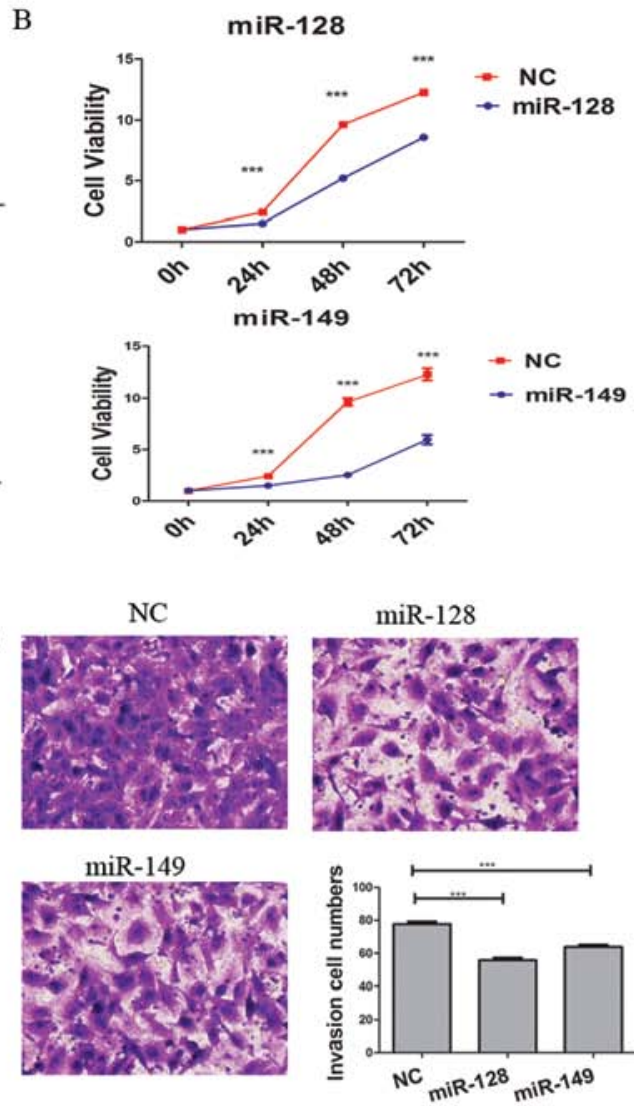

$\mathrm{F}$

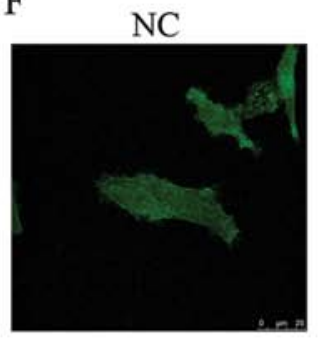

Si-Rap1B
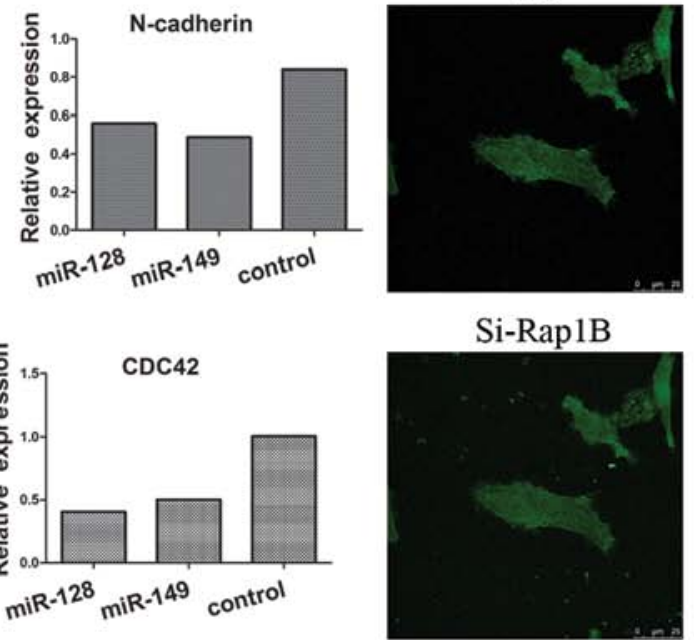

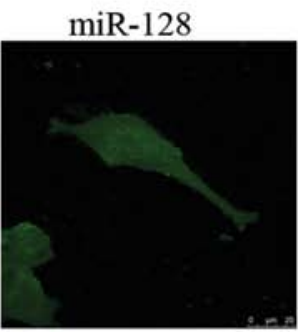

$\operatorname{miR}-149$

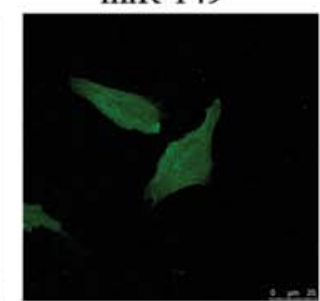

Figure 4. miR-128 and miR-149 inhibit the cellular proliferation and invasion by Rap1B-mediated actin cytoskeletal change. (A) Colony formation assay showing the reduced cellular colony formation after U251 cells were transfected with miR-128 or miR-149 mimics; ${ }^{* * * *} \mathrm{P}<0.001$. (B) MTT assay showing the reduced cellular proliferation after U251 cells were transfected with miR-128 or miR-149 mimics; ${ }^{* * *} \mathrm{P}<0.001$. (C) miR-128 or miR-149 inhibited tumor cell invasion as determined by wound closure assay in vitro. (D) Matrigel chamber invasion assay showing the reduced cellular invasion after U251 cells were transfected with miR-128 or miR-149 mimics; ${ }^{* * *} \mathrm{P}<0.001$. (E) Western blot analysis showing the protein expression of Cdc 42 , RhoA and N-cadherin after U251 cells were transfected with miR-128 or miR-149 mimics. (F) Phalloidin staining showing the expression and distribution of F-actin-rich cytoskeleton elements after U251 cells were transfected with miR-128 or miR-149 mimics or Si-Rap1B.

$(\mathrm{P}<0.001)$. MTT results indicated that miR-128 and miR-149 mimics also inhibited the proliferation of U251 cells at 72 and $96 \mathrm{~h}(\mathrm{P}<0.001)$ (Fig. 4B). The wound-healing assay showed that miR-128 and miR-149 inhibited the migration and motility of U251 cells. Overexpression of miR-128 and miR-149 using mimics clearly inhibited wound gap closure in a time-dependent manner (Fig. 4C). miR-128 and miR-149 mimics also inhibited the invasion of $\mathrm{U} 251$ cells at $48 \mathrm{~h}(\mathrm{P}<0.001)$ (Fig. 4D). It has been demonstrated that $\mathrm{TMZ}$ can inhibit the expression of Cdc42, RhoA and N-cadherin, and attenuate the expression of F-actin and its polarized distribution in U251 cells (18). In the present study, our results also indicated that miR-128 and miR-149 mimics inhibited the expression of Cdc42, RhoA and N-cadherin (Fig. 4E), suggesting that miR-128 and miR-149 
A
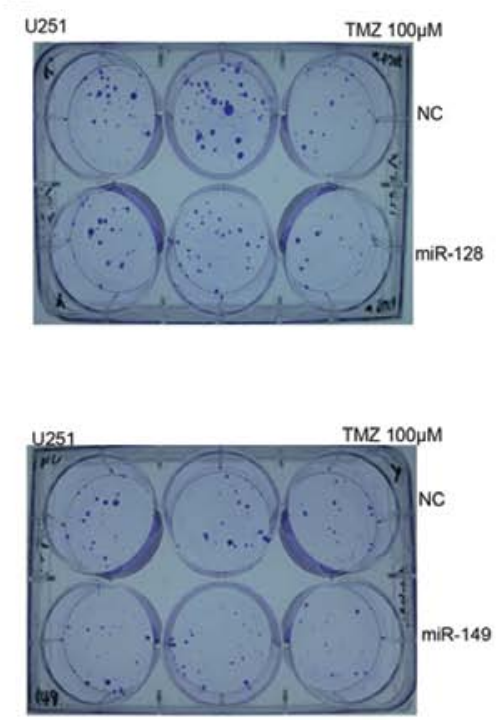

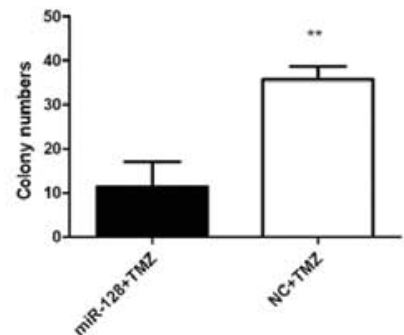

B

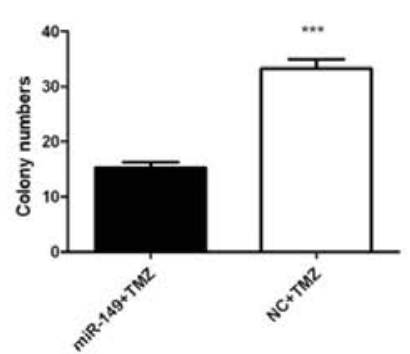

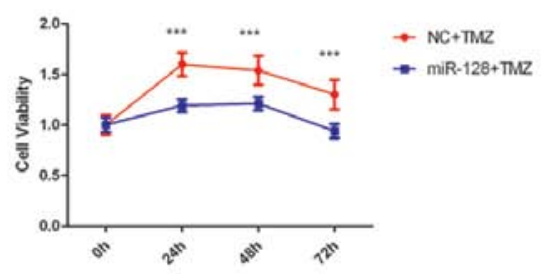

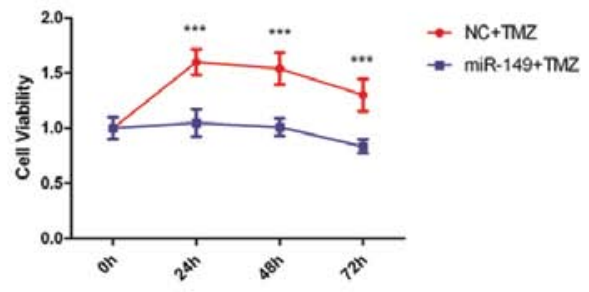

Figure 5. miR-128 and miR-149 enhance the chemosensitivity of conventional TMZ agent in glioblastoma cells. (A) Colony formation assay showing the reduced cellular colony formation when U251 cells were administered miR-128 or miR-149 mimics for $6 \mathrm{~h}$ and followed by subsequent treatment with $100 \mu \mathrm{M}$ TMZ; ${ }^{* *} \mathrm{P}<0.01 ;{ }^{* * *} \mathrm{P}<0.001$. (B) MTT assay showing the reduced cellular proliferation when U251 cells were administered miR-128 or miR-149 mimics for $6 \mathrm{~h}$ and followed by subsequent treatment with $100 \mu \mathrm{M} \mathrm{TMZ;}{ }^{* * *} \mathrm{P}<0.001$.

may be involved in cytoskeletal remodeling. FITC-labeled phalloidin staining showed that the expression of F-actin-rich cytoskeleton elements was strong and polarized at the edge of the U251 cells. However, the expression of F-actin was attenuated, and its polarized distribution died away when U251 cells were treated with miR-128 or miR-149 mimics and Si-Rap1B (Fig. 4F).

miR-128 and miR-149 increase the chemosensitivity of TMZ in glioblastoma cells. miR-128 and miR-149 inhibit the cellular proliferation, invasion and alter cytoskeletal remodeling by targeting inhibition of Rap1B. Therefore, we aimed to ascertain whether miR-128 and miR-149 enhance the chemosensitivity of temozolomide. The colony formation and MTT assays indicated that miR-128 and miR-149 each enhanced the killing effect of TMZ on U251 cells when cells were administered miR-128 and miR-149 for $6 \mathrm{~h}$, followed by subsequent treatment with $100 \mu \mathrm{M}$ TMZ (Fig. 5).

\section{Discussion}

GBM is characterized by high genetic heterogeneity and infiltrative brain invasion patterns, and aberrant miRNA expression has been associated with the hallmark malignant properties of GBM (31). microRNAs (miRNAs) are modulators of gene expression and play critical roles in various cellular processes. Several miRNAs have been characterized as tumor suppressors or oncogenes in cancer, and recent studies suggest that certain miRNAs are implicated in cytoskeletal remodeling and cell motility $(32,33)$. The cytoskeleton controls cell motility, invasion and adhesion. miR-23b is able to directly inhibit a number of genes implicated in cytoskeletal remodeling in breast cancer cells. Inhibition of miR-23b, using a specific sponge construct, was found to lead to an increase in cell migration and metastatic spread in vivo, identifying it as a metastatic suppressor microRNA. Clinically, low miR-23b expression correlates with the development of metastases in breast cancer patients (32). miR-30c was found to regulate invasion by targeting the cytoskeleton network genes encoding Twinfilin 1 (TWF1) and vimentin (VIM) in breast cancer cells (34). In the present study, we assessed the endogenous expression of miR-128 and miR-149 in 20 primary astrocytomas (grade I, 4 cases; grade II, 6 cases; grade III, 5 cases; and glioblastoma, 5 cases) and 8 normal brain tissues by quantitative real-time PCR. Our results indicated that the expression of miR-128 and miR-149 was downregulated in glioblastoma, and the expression of miR-149 gradually decreased with pathological progression in different grade astrocytomas. However, the expression of miR-128 was not different in WHO I, II, III grade astrocytomas and grade IV glioblastoma although there was a significant different in normal brain tissues and astrocytoma. These data revealed that aberrant expression of miR-149 was involved in the initiation and the malignant progression of astrocytomas, whereas, aberrant expression of miR-128 was associated with the initiation of the astrocytomas.

Our previous research found that Rap1B may also be a negative regulator of miR-128 and miR-149 by a docking study of miRNA/mRNA omics in human grade I-III astrocytomas (14). In the present study, we further confirmed that miR-128 and miR-149 have their own binding sites with Rap1B, and authenticated that Rap1B is a common target gene of miR-128 and miR-149, which negatively regulate the expression of Rap1B. RAP1 is a Ras-like GTPase that regulates several basic cellular functions, such as migration, adhesion and growth. RAP1A and RAP1B are two closely related isoforms of RAP1 (15). Rap1B, a small GTPase, is associated with the cytoskeleton during cell activation (35). Its downregulated expression is involved in the lysophosphatidic acid-induce inhibitory migration in glioma 
cells (36). Rho family GTPases, including Rac1, RhoA and cell division cycle 42 (Cdc42), participate in the regulation of adhesion, actin cytoskeletal reorganization and cell migration (37), and have been described as key regulators of cell migration (38). Several of the Rho GTPases shown to promote glioblastoma (GB) cell invasion have also been correlated with poor patient prognosis $(39,40)$. We further elucidated the biological function of miR-128 and miR-149. The results indicate that miR-128 and miR-149 mimics inhibited the proliferation and the invasion of U251 cells and also mediated inhibition of expression of Cdc42, RhoA and N-cadherin except for Rap1B. The overexpression of miR-128 and miR-149 or Rap1B siRNA could alter the F-actin cytoskeletal reorganization and its expression level. These functional studies suggest that miR-128 and miR-149 function as tumor-suppressor genes by Rap1B-mediated changes in the actin cytoskeleton and inhibition of cell adhesion and invasion and cell proliferation.

TMZ is the standard first-line agent for GBM. TMZ is compromised by its dependence for activity on DNA mismatch repair and the repair of the chemosensitive DNA lesion, $O^{6}$-methylguanine, by $O^{6}$-methylguanine-DNAmethyltransferase. The present study found that miR-128 and miR-149 inhibit the cellular proliferation, invasion and alter cytoskeletal remodeling by targeted inhibition of Rap1B. We then presumed that miR-128 and miR-149 enhance the chemosensitivity of temozolomide. Our data confirmed that miR-128 and miR-149 did increase the cellular inhibitory proliferation effects of TMZ in glioblastoma U251 cells as determined by colony formation and MTT assays, suggesting that the overexpression of miR-128 and miR-149 may enhance the chemosensitivity of TMZ.

In summary, the present study demonstrated that the expression of miR-128 and miR-149 was downregulated in glioblastoma; the lower expression of miR-128 and miR-149 contributed to astrocytoma tumorigenesis. Overexpression of miR-128 and miR-149 inhibited the invasion and proliferation of glioblastoma by targeting Rap1B-mediated cytoskeletal alteration and related molecules, such as $\mathrm{Cdc} 42$, RhoA and N-cadherin. miR-128 and miR-149 enhanced the chemosensitivity of human glioblastoma cells to TMZ. Therefore, restoration of miR-128 and miR-149 expression might provide a therapeutic strategy for GBM treatment. The combination of miR-128 and miR-149 mimics and TMZ could be an effective therapeutic strategy for suppressing the growth of GBM.

\section{Acknowledgements}

The present study was supported by grants from the National Science Foundation of China (81272297) and the Hunan Province Natural Sciences Foundations of China (11JJ1013).

\section{References}

1. Pichiorri F, Palmieri D, De Luca L, et al: In vivo NCL targeting affects breast cancer aggressiveness through miRNA regulation. J Exp Med 210: 951-968, 2013.

2. Pizzini S, Bisognin A, Mandruzzato S, et al: Impact of microRNAs on regulatory networks and pathways in human colorectal carcinogenesis and development of metastasis. BMC Genomics 14: 589, 2013.

3. Yang J, Zhao H, Xin Y and Fan L: MicroRNA-198 inhibits proliferation and induces apoptosis of lung cancer cells via targeting FGFR1. J Cell Biochem 115: 987-995, 2013.
4. Chen L, Zhang Y, Yang J, Hagan JP and Li M: Vertebrate animal models of glioma: understanding the mechanisms and developing new therapies. Biochim Biophys Acta 1836: 158-165, 2013.

5. Chou KN, Lin YC, Liu MY and Chang PY: Temozolomiderelated acute lymphoblastic leukemia with translocation $(4 ; 11)$ (q21;q23) in a glioblastoma patient. J Clin Neurosci 21: 701-704 2013.

6. Danson SJ and Middleton MR: Temozolomide: a novel oral alkylating agent. Expert Rev Anticancer Ther 1: 13-19, 2001.

7. Stupp R, Gander M, Leyvraz S and Newlands E: Current and future developments in the use of temozolomide for the treatment of brain tumours. Lancet Oncol 2: 552-560, 2001.

8. Wong STS, Zhang XQ, Zhuang JTF, Chan HL, Li CH and Leung GKK: MicroRNA-21 inhibition enhances in vitro chemosensitivity of temozolomide-resistant glioblastoma cells. Anticancer Res 32: 2835-2841, 2012.

9. Shi L, Chen JA, Yang JA, Pan TH, Zhang SG and Wang ZM: MiR-21 protected human glioblastoma U87MG cells from chemotherapeutic drug temozolomide induced apoptosis by decreasing Bax/Bcl-2 ratio and caspase-3 activity. Brain Res 1352: 255-264, 2010.

10. Qian XM, Ren Y, Shi ZD, et al: Sequence-dependent synergistic inhibition of human glioma cell lines by combined temozolomide and miR-21 inhibitor gene therapy. Mol Pharm 9: 2636-2645, 2012.

11. Ujifuku K, Mitsutake N, Takakura S, et al: miR-195, miR-455-3p and $\mathrm{miR}-10 \mathrm{a}^{*}$ are implicated in acquired temozolomide resistance in glioblastoma multiforme cells. Cancer Lett 296: 241-248, 2010.

12. Slaby O, Lakomy R, Fadrus P, et al: MicroRNA-181 family predicts response to concomitant chemoradiotherapy with temozolomide in glioblastoma patients. Neoplasma 57: 264-269, 2010.

13. Shi L, Zhang SG, Feng K, et al: MicroRNA-125b-2 confers human glioblastoma stem cells resistance to temozolomide through the mitochondrial pathway of apoptosis. Int J Oncol 40: 119-129, 2012.

14. Li D, Chen P, Li XY, et al: Grade-specific expression profiles of miRNAs/mRNAs and docking study in human grade I-III astrocytomas. OMICS 15: 673-682, 2011.

15. Wittchen ES, Aghajanian A and Burridge K: Isoform-specific differences between Rap1 A and Rap1B GTPases in the formation of endothelial cell junctions. Small GTPases 2: 65-76, 2011.

16. Nuovo GJ: In situ detection of precursor and mature microRNAs in paraffin embedded, formalin fixed tissues and cell preparations. Methods 44: 39-46, 2008.

17. Evangelisti C, Florian MC, Massimi I, et al: MiR-128 up-regulation inhibits Reelin and DCX expression and reduces neuroblastoma cell motility and invasiveness. FASEB J 23: 4276-4287, 2009.

18. Zhang Y, Chao T, Li R, et al: MicroRNA-128 inhibits glioma cells proliferation by targeting transcription factor E2F3a. J Mol Med 87: 43-51, 2009.

19. Costa PM, Cardoso AL, Nobrega C, et al: MicroRNA-21 silencing enhances the cytotoxic effect of the antiangiogenic drug sunitinib in glioblastoma. Hum Mol Genet 22: 904-918, 2013.

20. Khan AP, Poisson LM, Bhat VB, et al: Quantitative proteomic profiling of prostate cancer reveals a role for miR-128 in prostate cancer. Mol Cell Proteomics 9: 298-312, 2010.

21. Weiss GJ, Bemis LT, Nakajima E, et al: EGFR regulation by microRNA in lung cancer: correlation with clinical response and survival to gefitinib and EGFR expression in cell lines. Ann Oncol 19: 1053-1059, 2008

22. Kotani A, Ha D, Schotte D, den Boer ML, Armstrong SA and Lodish HF: A novel mutation in the miR-128b gene reduces miRNA processing and leads to glucocorticoid resistance of MLL-AF4 acute lymphocytic leukemia cells. Cell Cycle 9: 1037-1042, 2010

23. Zhu Y, Yu F, Jiao Y, et al: Reduced miR-128 in breast tumorinitiating cells induces chemotherapeutic resistance via Bmi-1 and ABCC5. Clin Cancer Res 17: 7105-7115, 2011.

24. Shi ZM, Wang J, Yan Z, et al: miR-128 inhibits tumor growth and angiogenesis by targeting p70S6K1. PLoS One 7: e32709, 2012.

25. Lin RJ, Lin YC and Yu AL: miR-149* induces apoptosis by inhibiting Akt1 and E2F1 in human cancer cells. Mol Carcinog 49: 719-727, 2010.

26. Jin L, Hu WL, Jiang CC, et al: MicroRNA-149*, a p53-responsive microRNA, functions as an oncogenic regulator in human melanoma. Proc Natl Acad Sci USA 108: 15840-15845, 2011.

27. Wang Y, Zheng XS, Zhang ZY, et al: MicroRNA-149 inhibits proliferation and cell cycle progression through the targeting of ZBTB2 in human gastric cancer. PLoS One 7: e41693, 2012. 
28. Tu HF, Liu CJ, Chang CL, et al: The association between genetic polymorphism and the processing efficiency of miR-149 affects the prognosis of patients with head and neck squamous cell carcinoma. PLoS One 7: e51606, 2012.

29. Pan SJ, Zhan SK, Pei BG, Sun QF, Bian LG and Sun BM: MicroRNA-149 inhibits proliferation and invasion of glioma cells via blockade of AKT1 signaling. Int J Immunopathol Pharmacol 25: 871-881, 2012

30. Tang HL, Wang ZY, Liu XP, et al: LRRC4 inhibits glioma cell growth and invasion through a miR-185-dependent pathway. Curr Cancer Drug Targets 12: 1032-1042, 2012.

31. Guo Y, Yan KP, Fang JS, Qu Q, Zhou M and Chen FH: Let-7b expression determines response to chemotherapy through the regulation of cyclin D1 in glioblastoma. J Exp Clin Cancer Res 32: 41, 2013.

32. Pellegrino L, Stebbing J, Braga VM, et al: miR-23b regulates cytoskeletal remodeling, motility and metastasis by directly targeting multiple transcripts. Nucleic Acids Res 41: 5400-5412, 2013

33. Avellino R, Carrella S, Pirozzi M, et al: miR-204 targeting of Ankrd13A controls both mesenchymal neural crest and lens cell migration. PLoS One 8: e61099, 2013.

34. Bockhorn J, Yee K, Chang YF, et al: MicroRNA-30c targets cytoskeleton genes involved in breast cancer cell invasion. Breast Cancer Res Treat 137: 373-382, 2013.
35. Bertoni A, Tadokoro S, Eto K, et al: Relationships between Rap1b, affinity modulation of integrin $\alpha \operatorname{IIb} \beta 3$, and the actin cytoskeleton. J Biol Chem 277: 25715-25721, 2002.

36. Malchinkhuu E, Sato K, Maehama T, et al: Role of Rap1B and tumor suppressor PTEN in the negative regulation of lysophosphatidic acid-induced migration by isoproterenol in glioma cells. Mol Biol Cell 20: 5156-5165, 2009.

37. Vega FM and Ridley AJ: Rho GTPases in cancer cell biology. FEBS Lett 582: 2093-2101, 2008.

38. de Toledo M, Anguille C, Roger L, Roux P and Gadea G: Cooperative anti-invasive effect of $\mathrm{Cdc} 42 / \mathrm{Racl}$ activation and ROCK inhibition in SW620 colorectal cancer cells with elevated blebbing activity. PLoS One 7: e48344, 2012.

39. Feng H, Hu B, Liu KW, et al: Activation of Rac1 by Src-dependent phosphorylation of Dock $180^{\mathrm{Y} 1811}$ mediates PDGFR $\alpha$-stimulated glioma tumorigenesis in mice and humans. J Clin Invest 121: 4670-4684, 2011.

40. Fortin Ensign SP, Mathews IT, Eschbacher JM, Loftus JC, Symons MH and Tran NL: The Src homology 3 domaincontaining guanine nucleotide exchange factor is overexpressed in high-grade gliomas and promotes tumor necrosis factor-like weak inducer of apoptosis-fibroblast growth factor-inducible 14-induced cell migration and invasion via tumor necrosis factor receptor-associated factor 2. J Biol Chem 288: 21887-21897, 2013. 